\title{
TREATED AND UNTREATED WASTEWATER IMPARTS MORPHOLOGICAL CHANGES TO SCENTED ROSA SPECIES IN PERI-URBAN AREA
}

\author{
Muhammad Ahsan 1,", Adnan Younis², Atif Riaz², Muhammad Jafar Jaskani², Muhammad \\ Qasim², Mansoor Hameed $^{3}$, Aasma Tufail ${ }^{4}$, Muhammad Nafees ${ }^{1}$, Haider Abbas ${ }^{5}$ and \\ Usman Tariq ${ }^{6}$
}

\author{
${ }^{1}$ Department of Horticultural Sciences, University College of Agriculture and Environmental Sciences, The Islamia \\ University of Bahawalpur, Pakistan; ${ }^{2}$ Institute of Horticultural Sciences, University of Agriculture Faisalabad, \\ Pakistan; ${ }^{3}$ Department of Botany, University of Agriculture Faisalabad, Pakistan; ${ }^{4}$ Department of Botany, Division of \\ Science and Technology, University of Education Lahore, Pakistan; ${ }^{5}$ Department of Agriculture and Agribusiness \\ Management, University of Karachi, Karachi, Pakistan ${ }^{6}$ College of Agriculture, Bahadur sub-campus Layyah, \\ Bahaudin Zakariya University, Multan, Pakistan \\ *Corresponding author's e-mail: ahsan2065@gmail.com; ahsan.horti@iub.edu.pk
}

\begin{abstract}
An experiment was conducted to assess the effects of treated and untreated waste water on morphological characteristics of four widely cultivated fragrant Rosa species of Pakistan during 2012-2013. Experiment was designed according to randomized complete block design with two factor factorial arrangement. One treatment factor was Rosa species and other was irrigation source. All minerals and chemicals present in canal water and treated waste water were in permissible level, whereas untreated waste water contained higher values of EC, biological oxygen demand (BOD), chemical oxygen demand (COD) and heavy metals like $\mathrm{Cd}, \mathrm{Co}, \mathrm{Cu}, \mathrm{Pb}$. Results showed that maximum plant height and number of leaves per branch was recorded in $R$. bourboniana and $R$. damascena respectively under treated waste water and minimum height was found in $R$. centifolia under untreated waste water whereas highest leaf area was recorded in $R$. damascena under canal water and treated waste water during $1^{\text {st }}$ and $2^{\text {nd }}$ year of experiment respectively. $R$. Gruss-an-Teplitz was dominant species for floral characteristics like minimum days to flower emergence, flower diameter, number of flowers plant ${ }^{-1}$ and number of petals flower ${ }^{-1}$ under treated waste water whereas thickest flower bud was recorded under untreated waste water. Due to high load of contaminants, untreated waste water was responsible to produced minimum flower numbers in all species. In present study, $R$. Gruss-an-Teplitz was found to be most resistant and suitable rose species to cultivate in peri-urban areas under treated and untreated waste water and it is strongly recommended that untreated waste water must be treated to some extent before application to roses.
\end{abstract}

Keywords: Floral growth, heavy metals, water quality, roses, vegetative characteristics

\section{INTRODUCTION}

It is centuries old practice to use municipal waste water in agriculture, which has lately gained renewed consideration in many areas of the world due to the increasing shortage of irrigation water (Khaleel et al., 2013; Kurian, 2017). Increasing the use of treated and untreated waste water in periurban agriculture and even in distant rural areas where its usage originates, increases economic activity and improves the livelihood of poor growers but modifies the quality of environment (Abu Qdais and Al-Widyan, 2016; Murtaza et al., 2010; Talal et al., 2014). The efficient use of municipal sewage water can efficiently increase water resources for irrigation and may prove to be a bonus for agricultural production and for this purpose, reclaimed municipal waste water can be an alternative source of nutrients and water for horticultural and nursery crop production since nutrients are available in a usable form and, in general, do not need any supplementary energy input to make them accessible to plants
(Asgharipour and Azizmoghaddam, 2012; Farahat and Linderholm, 2013).

As agriculture sector in Pakistan is dominated by the farmers of small land holdings and production of conventional crops is no more profitable, therefore the shift from conventional to high value crops and introduction of new alternative crops like flowers cultivation is increasing overtime. In Pakistan, the most important floricultural crops are rose, jasmine, gladiolus, tuberose, carnation, iris, narcissus, lilies and gerbra (Riaz et al., 2008). Among these crops; rose is first ranked high value floricultural crop. It is woody perennial flowering plant of famous plant family Rosaceae. Economically roses are considered as highly viable crop with a share of $65 \%$ of total floricultural trade in under developed countries of the world (Janko and Alemu, 2014). There are four important species of roses that are grown for essential oil production that is used in perfumes, medicine, cosmetics and many other products. Top ranked is $R$. damascena which is extensively cultivated in Bulgaria (70-80\%), China, Turkey and India 
while second one is $R$. centifolia, commonly grown in France, Egypt and Morocco (Nasir et al., 2007; Farooq et al., 2016). Later come $R$. bourboniana and $R$. Gruss-an-Teplitz which were introduced in France and China respectively (Laurie and Ries, 1950).

In peri-urban areas of large cities like Faisalabad, both untreated and treated forms of waste water are used for production of vegetables (Hussain et al., 2006). These vegetables are enriched with heavy metals in waste water that causes human health impairment of humans in peri-urban and urban areas. Ornamental crops can be more appropriate for growing with treated and untreated waste water as they are not consumed and health issues are lesser. Keeping in view the effects of treated and untreated waste water, a field experiment was carried out to determine the morphological characteristics of four scented and oil bearing species of high value floricultural crop of roses under raw sewage untreated and treated waste water in peri-urban area.

\section{MATERIALS AND METHODS}

Experimental site, soil analysis and experimental treatments: The experiment was carried out at the Agronomy Research Area of University of Agriculture, Faisalabad $\left(31^{\circ} 25^{\prime} \mathrm{N}, 73^{\circ} 09^{\prime} \mathrm{E}\right.$ and altitude of $300 \mathrm{~m}$ above mean sea level) Pakistan from $2^{\text {nd }}$ January 2012 to $30^{\text {th }}$ December, 2013. Soil of this experimental area is clay loam which collects sewage waste water from the students living hostels of University of Agriculture Faisalabad and canal water from main canal of the city. Before start of experiment, sixteen soil samples were randomly collected at the depth of 15 and 30 $\mathrm{cm}$. Composite soil samples were analyzed according to the standard procedures (U.S. Silinity Laboratory Staff, 1954) and results are presented in Table 1.

There were two treatment factors of this experiment i.e. irrigation water (canal water, treated waste water and untreated waste water) and Rosa species ( $R$. centifolia, $R$. damascena, $R$. bourboniana and $R$. Gruss-an-Teplitz).

Water treatment and analysis: In this experiment, untreated waste water was treated by natural purification process as discussed by Kiziloglu et al. (2008) to improve its physical and chemical quality using conventional method (Pescod, 1992) in three large plastic tanks of 1500 gallons water storage capacity, in three step process i.e. primary, secondary and tertiary treatment (Pescod, 1992). Physio-chemical properties of all irrigation water types were determined by standard methods of waste water examination proposed by Eaton et al., (2005) and all heavy metals and some nutrients like $\mathrm{P}, \mathrm{K}, \mathrm{Na}$ and $\mathrm{Ca}$ concentration was determined with the help of inductively couples plasma (ICP-OES) (Optima 2100DV Perkin Elmer) at Nuclear Institute of Agriculture and Biology (NIAB) Faisalabad, Pakistan.

Rosa species and morphological characteristics: Two years old cuttings of fragrant Rosa species were planted during first week of January 2012 and irrigated by canal water, treated and untreated waste water. Data regarding vegetative parameters (plant height, number of leaves per branch, leaf area) and floral characteristics (days to flower emergence, bud and flower diameter, number of flowers per plant, number of petals per flower) were studied.

Experimental design and statistical analysis: Experimental treatments were set according to randomized complete block design (RCBD) and there were 15 plants of every single species in each treatment with total of 180 plants in single replication which were repeated thrice. Data collected for all parameters were analyzed by performing Fisher`s analysis of variance technique (ANOVA) using Statistica soft 5.5 and treatment means were compared according to least significant difference (LSD) test at 5\% level of probability (Steel et al., 1997).

\section{RESULTS}

Physio-chemical analysis of canal water, treated and untreated waste water was carried out (Table 2) before the experiment. Data of water analysis showed that EC of untreated waste water was above the standard limit values set by International Irrigation Water Quality Standards and National Environmental Quality Standards for municipal waste waters of Pakistan. Untreated waste water also contained higher BOD, COD, heavy metal $(\mathrm{Cd}, \mathrm{Pb}, \mathrm{Co}, \mathrm{Cu})$, $\mathrm{Na}$ and $\mathrm{N}$ level while treated waste water and canal water contained all physical and chemical values within permissible limits.

Effect of treated and untreated wastewaters on vegetative growth parameters:

Plant height $(\mathbf{c m})$ : The results showed that treated waste water produced taller rose plants compared to canal water and untreated waste water in 2012 where maximum plant height $(148.10 \mathrm{~cm})$ was observed in $R$. bourboniana while minimum height was found in $R$. damascena $(83.23 \mathrm{~cm})$ under untreated waste water. In $2013, R$. bourboniana $(137.20 \mathrm{~cm})$ under untreated waste water produced maximum height and minimum height was found in $R$. centifolia $(66.80 \mathrm{~cm})$ under untreated waste water. It was observed that all Rosa species

Table 1. Soil composition before experiment.

\begin{tabular}{llccccccccccc}
\hline $\begin{array}{l}\text { Soil } \\
\text { characteristics }\end{array}$ & Texture & $\mathbf{p H}$ & $\mathbf{E C}$ & $\mathbf{O M}$ & $\mathbf{N}$ & $\mathbf{P}$ & $\mathbf{K}$ & $\mathbf{P b}$ & $\begin{array}{c}\mathbf{C d} \\
(\%)\end{array}$ & $\begin{array}{c}\mathbf{N i} \\
(\%)\end{array}$ & $\begin{array}{c}\mathbf{Z n} \\
(\mathrm{ppm})\end{array}$ & $\begin{array}{c}\mathbf{C u} \\
(\mathrm{ppm})\end{array}$ \\
\hline $00-15 \mathrm{~cm}$ & Clay & 8.20 & 2.54 & 1.12 & 0.041 & 10.50 & 194 & 3.16 & 0.04 & 0.36 & 5.28 & 3.04 \\
$16-30 \mathrm{~cm}$ & loam soil & 8.20 & 2.49 & 1.18 & 0.041 & 9.50 & 134 & 3.32 & 0.05 & 0.34 & 3.60 & 2.30 \\
IASS & & $4-8.5$ & 4.00 & $>0.86$ & --- & $>7$ & $>80$ & 500 & 1.0 & 20 & 250 & 100 \\
\hline
\end{tabular}

$\mathrm{EC}=$ Electrical conductivitv. OM= Organic matter. "IASS= International Agricultural Soil Standards: Source: Allowav (1990) 
Table 2. Composition of canal water, treated and untreated waste water.

\begin{tabular}{|c|c|c|c|c|}
\hline Parameters & Canal Water & Treated Water & Untreated Water & IIWQS/NEQS $^{* * *}$ \\
\hline $\mathrm{EC}(\mu \mathrm{S} / \mathrm{L})$ & 1.13 & 1.44 & 2.11 & $1.5^{\dagger}$ \\
\hline $\mathrm{pH}$ & 7.42 & 7.58 & 8.31 & $6-9.2^{\dagger}$ \\
\hline Color & ---- & Rust Brown & Greyish & -- \\
\hline Turbidity & 43 & 29.12 & 155 & -- \\
\hline Hardness (mg/L) & 184 & 416 & 536 & -- \\
\hline $\mathrm{DO}(\mathrm{mg} / \mathrm{L})$ & 4 & 2.38 & 1.36 & -- \\
\hline $\mathrm{BOD}(\mathrm{mg} / \mathrm{L})$ & --- & 267 & 432 & $300^{\dagger}$ \\
\hline $\mathrm{COD}(\mathrm{mg} / \mathrm{L})$ & --- & 481 & 669 & $500^{\dagger}$ \\
\hline TDS (mg/L) & 218 & 1281 & 1678 & $2500^{\dagger}$ \\
\hline $\mathrm{SS}(\mathrm{mg} / \mathrm{L})$ & 0.9 & 0.15 & 1.1 & -- \\
\hline Total Solids (mg/L) & 218 & 982 & 1372 & -- \\
\hline $\mathrm{TSS}(\mathrm{mg} / \mathrm{L})$ & 24 & 63 & 194 & $400^{\dagger}$ \\
\hline Chlorides (mg/L) & 138 & 290 & 436 & $1000^{\dagger}$ \\
\hline Cadmium (mg/L) & 0.001 & 0.01 & 0.013 & $0.01^{\dagger \dagger}$ \\
\hline Nickel (mg/L) & 0.10 & 0.08 & 0.12 & $0.2^{\dagger \dagger}$ \\
\hline Arsenic (mg/L) & ND & 0.004 & 0.005 & $0.1^{\dagger \dagger}$ \\
\hline Zinc (mg/L) & 0.18 & 2.62 & 3.48 & $5.0^{\dagger \dagger}$ \\
\hline Potassium (mg/L) & 30.41 & 17.61 & 40.73 & -- \\
\hline Lead (mg/L) & 0.021 & 0.42 & 0.66 & $0.5^{\dagger}$ \\
\hline Iron $(\mathrm{mg} / \mathrm{L})$ & 0.32 & 3.47 & 4.82 & $5.0^{\dagger \dagger}$ \\
\hline Cobalt (mg/L) & 0.17 & 0.029 & 0.079 & $0.05^{\dagger}$ \\
\hline Copper (mg/L) & 0.05 & 0.13 & 0.24 & $0.2^{\dagger \dagger}$ \\
\hline Chromium (mg/L) & 0.04 & 0.067 & 0.093 & $0.1^{\dagger \dagger}$ \\
\hline Calcium (mg/L) & 28.1 & 39.72 & 54.29 & $230^{\dagger \dagger}$ \\
\hline Sodium $(\mathrm{mg} / \mathrm{L})$ & 36.47 & 178.23 & 252.77 & $230^{\dagger \dagger}$ \\
\hline Magnesium (mg/L) & 30 & 47 & 63 & $100^{\dagger \dagger}$ \\
\hline Phosphorus (mg/L) & 0.39 & 1.76 & 2.49 & $15^{\dagger}$ \\
\hline Total Nitrogen (mg/L) & 4 & 5.72 & 8.0 & $5.0^{\dagger}$ \\
\hline
\end{tabular}

**IIWQS: International Irrigation Water Quality Standards; NEQS: National Environmental Quality Standards for municipal wastewater of Pakistan; †: Standard value of NEQS; ††: Standard value of IIWQS; EC: Electrical conductivity; DO: Dissolved Oxygen; BOD: Biological Oxygen Demand; COD: Chemical Oxygen Demand; TDS: Total Dissolved Solids; SS: Settle able Solids; TSS: Total Suspended Solids. NEQS source: Anon., (2007); WHO, (1989).

Table 3. Vegetative growth characteristics of Rosa species under different irrigation treatments.

\begin{tabular}{|c|c|c|c|c|c|c|c|c|}
\hline \multicolumn{9}{|c|}{ Plant height (cm) } \\
\hline \multirow{2}{*}{$\begin{array}{l}\text { Rosa } \\
\text { species }\end{array}$} & \multicolumn{4}{|c|}{2012} & \multicolumn{4}{|c|}{2013} \\
\hline & CW & TW & UTW & Mean & CW & TW & UTW & Mean \\
\hline R.B. & $120.40 \pm 8.2^{\mathrm{bc}}$ & $148.10 \pm 6.4^{\mathrm{a}}$ & $135.10 \pm 9.8^{\mathrm{ab}}$ & $134.53^{\mathrm{a}}$ & $131.33 \pm 7.3^{\mathrm{a}}$ & $132.60 \pm 5.1^{\mathrm{a}}$ & $137.20 \pm 3.3^{\mathrm{a}}$ & $133.71^{\mathrm{a}}$ \\
\hline R.C & $93.63 \pm 6.8^{\mathrm{efg}}$ & $100.77 \pm 3.1^{\text {def }}$ & $85.83 \pm 4.8^{\mathrm{fg}}$ & $93.41^{\mathrm{c}}$ & $76.17 \pm 2.9^{\text {ef }}$ & $98.03 \pm 4.7^{\mathrm{cd}}$ & $66.80 \pm 1.9^{f}$ & $80.33^{\mathrm{d}}$ \\
\hline G.T & $109.93 \pm 3.9^{\mathrm{cd}}$ & $113.50 \pm 2.8^{\mathrm{cd}}$ & $104.23 \pm 2.7^{\mathrm{de}}$ & $109.22^{b}$ & $112.93 \pm 6.8^{\mathrm{b}}$ & $106.60 \pm 4.5^{\mathrm{bc}}$ & $103.93 \pm 4.9^{\mathrm{bc}}$ & $107.82^{\mathrm{b}}$ \\
\hline R.D & $100.77 \pm 4.7^{\text {def }}$ & $114.13 \pm 2.3^{\mathrm{cd}}$ & $83.27 \pm 5.5^{\mathrm{g}}$ & $99.39^{c}$ & $84.43 \pm 2.8^{\mathrm{de}}$ & $101.17 \pm 5.1^{\mathrm{bc}}$ & $81.37 \pm 4.1^{\mathrm{e}}$ & $88.99^{c}$ \\
\hline Average & $106.18^{b}$ & $119.12^{\mathrm{a}}$ & $102.11^{\mathrm{b}}$ & & $101.22^{\mathrm{b}}$ & $109.60^{\mathrm{a}}$ & $97.33^{\mathrm{b}}$ & \\
\hline \multicolumn{9}{|c|}{ Number of leaves per branch } \\
\hline R.B. & $28.95 \pm 1.0^{\mathrm{e}}$ & $33.66 \pm 0.9^{a b c}$ & $32.9 \pm 1.8^{\mathrm{bcd}}$ & $31.84^{\mathrm{b}}$ & $29.42 \pm 1.3^{\text {cde }}$ & $29.14 \pm 1.1^{\text {cde }}$ & $30.57 \pm 1.1^{\mathrm{bcd}}$ & $29.71^{\mathrm{b}}$ \\
\hline R.C & $29.00 \pm 1.1^{\mathrm{e}}$ & $29.81 \pm 1.4^{\mathrm{de}}$ & $29.14 \pm 1.2^{\mathrm{e}}$ & $29.32^{\mathrm{c}}$ & $26.41 \pm 1.1^{\mathrm{e}}$ & $27.28 \pm 0.9^{\mathrm{e}}$ & $27.19 \pm 1.1^{\mathrm{e}}$ & $26.96^{\mathrm{c}}$ \\
\hline G.T & $27.33 \pm 1.1^{\mathrm{e}}$ & $30.38 \pm 1.1^{\mathrm{cde}}$ & $29.04 \pm 0.9^{\mathrm{e}}$ & $28.92^{\mathrm{c}}$ & $27.9 \pm 0.9^{\mathrm{de}}$ & $32.19 \pm 1.6^{\mathrm{abc}}$ & $29.42 \pm 0.9^{\text {cde }}$ & $29.84^{\mathrm{c}}$ \\
\hline R.D & $32.85 \pm 1.0^{\mathrm{bcd}}$ & $36.33 \pm 1.1^{\mathrm{a}}$ & $35.09 \pm 1.0^{\mathrm{ab}}$ & $34.76^{\mathrm{a}}$ & $33.19 \pm 1.0^{\mathrm{ab}}$ & $33.52 \pm 0.7^{\mathrm{ab}}$ & $33.95 \pm 0.7^{\mathrm{a}}$ & $33.55^{\mathrm{a}}$ \\
\hline Average & $29.53^{b}$ & $32.54^{\mathrm{a}}$ & $31.54^{\mathrm{a}}$ & & $29.23^{\mathrm{a}}$ & $30.53^{\mathrm{a}}$ & $30.28^{\mathrm{a}}$ & \\
\hline \multicolumn{9}{|c|}{ Leaf area $\left(\mathrm{cm}^{2}\right)$} \\
\hline R.B. & $87.77 \pm 5.4^{\mathrm{fg}}$ & $83.42 \pm 4.3^{\mathrm{g}}$ & $92.2 \pm 5.2^{\mathrm{efg}}$ & $87.80^{c}$ & $86.43 \pm 3.8^{\mathrm{gh}}$ & $89.33 \pm 4.6^{\text {fgh }}$ & $83.26 \pm 5.2^{\mathrm{h}}$ & $86.34^{\mathrm{c}}$ \\
\hline R.C & $120.52 \pm 5.4^{\mathrm{b}}$ & $108.16 \pm 6.5^{\mathrm{bcd}}$ & $101.77 \pm 4.3^{\mathrm{def}}$ & $110.15^{b}$ & $102.85 \pm 2.9^{\text {cde }}$ & $115.65 \pm 4.7^{\mathrm{abc}}$ & $98.93 \pm 4.9^{\mathrm{efg}}$ & $105.81^{\mathrm{b}}$ \\
\hline G.T & $103.56 \pm 6.7^{\text {cde }}$ & $101.93 \pm 3.9^{\text {def }}$ & $107.61 \pm 4.4^{\text {bcd }}$ & $104.37^{\mathrm{b}}$ & $108.21 \pm 3.8^{\mathrm{b}-\mathrm{e}}$ & $101.49 \pm 3.7^{\mathrm{def}}$ & $105.68 \pm 4.4^{\text {cde }}$ & $105.13^{b}$ \\
\hline R.D & $135.63 \pm 4.3^{\mathrm{a}}$ & $117.42 \pm 6.8^{\mathrm{bc}}$ & $119.43 \pm 4.8^{\mathrm{b}}$ & $124.16^{\mathrm{a}}$ & $120.02 \pm 2.8^{\mathrm{ab}}$ & $125.22 \pm 6.3^{\mathrm{a}}$ & $114.8 \pm 5.4^{\mathrm{abcd}}$ & $120.01^{\mathrm{a}}$ \\
\hline Average & $111.87^{\mathrm{a}}$ & $102.73^{\mathrm{b}}$ & $105.25^{\mathrm{ab}}$ & & $104.37^{\mathrm{ab}}$ & $107.92^{\mathrm{a}}$ & $100.67^{\mathrm{b}}$ & \\
\hline
\end{tabular}

Treatments sharing similar statistical letters are significantly not different from each other. $\mathrm{CW}=\mathrm{Canal}$ water; $\mathrm{TW}=\mathrm{Treated}$ waste water;

$\mathrm{UTW}=$ Untreated waste water. $\mathrm{RB}=$ Rosa bourboniana $; \mathrm{RC}=$ Rosa centifolia $; \mathrm{GT}=$ Gruss-an-Teplitz $; \mathrm{RD}=$ Rosa damascena .

responded positively to treated waste water whereas plant height was reduced in untreated waste water (Table 3).
Number of leaves per branch: Maximum leaves banch ${ }^{-1}$ was recorded in $R$. damascena (36.33) produced under treated 
waste water followed by same Rosa species in untreated waste water (35.09) in 2012 while minimum leaves were calculated in $R$. Gruss-an-Teplitz (27.33) under canal water treatment. During 2013, $R$. damascena (33.95) under untreated waste water produced maximum and $R$. centifolia (26.42) produced minimum number of leaves per branch under canal water treatment (Table 3).

Leaf area $\left(\mathrm{cm}^{2}\right)$ : Data showed that maximum leaf area was found in $R$. damascena $\left(135.63 \mathrm{~cm}^{2}\right)$ under canal water while $R$. bourboniana under treated waste water treatment produced minimum value $\left(83.42 \mathrm{~cm}^{2}\right)$ during 2012 . Leaf area of $R$. centifolia and $R$. Gruss-an-Teplitz was in medium range than values of $R$. damascena and $R$. bourboniana. In 2013, $R$. damascena $\left(125.22 \mathrm{~cm}^{2}\right)$ under treated waste water produced highest value while untreated waste water in $R$. bourboniana $\left(83.26 \mathrm{~cm}^{2}\right)$ produced lowest value of leaf area.

Effect of treated and untreated waste waters on floral growth characteristics:

Days to flower emergence: The results of days to first flower appearance are presented (Table 4) which showed that during $R$. Gruss-an-Teplitz in 2012 took 25.42 days to produce first flower under treated waste during $1^{\text {st }}$ year of experiment. Maximum days (76.57) were taken by $R$. damascena in treated waste water treatment. During $2^{\text {nd }}$ year, $R$. Gruss-anTeplitz produced flower after 25.28 days under treated waste water that is lowest number of days to produce first flower whereas $R$. damascena (71.95) under canal water treatment took highest number of days to produce first flower during 2013 among counterparts.

Flower bud diameter $(\mathbf{m m})$ : The highest value of flower bud diameter was measured in $R$. Gruss-an-Teplitz $(12.55 \mathrm{~mm})$ under untreated waste water while minimum value was recorded in $R$. damascena $(9.37 \mathrm{~mm})$ under untreated waste water irrigation in 2012. Highest bud diameter values in $R$. centifolia and $R$. bourboniana were $10.87 \mathrm{~mm}$ and $10.23 \mathrm{~mm}$ respectively in treated and untreated waste water treatment respectively. In 2013, minimum flower bud diameter was found for $R$. damascena $(8.98 \mathrm{~mm})$ under untreated waste

Table 4. Floral characteristics of Rosa species under different irrigation treatments.

\begin{tabular}{|c|c|c|c|c|c|c|c|c|}
\hline \multicolumn{9}{|c|}{ Days to flower emergence } \\
\hline \multirow{2}{*}{$\begin{array}{l}\text { Rosa } \\
\text { species }\end{array}$} & \multicolumn{4}{|c|}{2012} & \multicolumn{4}{|c|}{2013} \\
\hline & CW & TW & UTW & Mean & CW & TW & UTW & Mean \\
\hline R.B. & $72.57 \pm 3.1^{\mathrm{a}}$ & $68.81 \pm 3.6^{\mathrm{a}}$ & $69.76 \pm 5.2^{\mathrm{a}}$ & $70.38^{\mathrm{a}}$ & $71.14 \pm 4.2^{\mathrm{a}}$ & $68.9 \pm 4.5^{\mathrm{a}}$ & $65.38 \pm 2.8^{\mathrm{a}}$ & $68.47^{\mathrm{a}}$ \\
\hline R.C & $38.00 \pm 2.9^{\mathrm{bc}}$ & $42.52 \pm 5.3^{\mathrm{b}}$ & $44.85 \pm 3.3^{\mathrm{b}}$ & $41.79^{b}$ & $46.09 \pm 2.8^{\mathrm{b}}$ & $40.52 \pm 3.1^{\mathrm{b}}$ & $46.19 \pm 1 . .6^{b}$ & $44.27^{\mathrm{b}}$ \\
\hline G.T & $27.09 \pm 3.6^{\mathrm{cd}}$ & $25.42 \pm 3.5^{\mathrm{d}}$ & $27.52 \pm 1.9^{\mathrm{d}}$ & $26.68^{b}$ & $27.14 \pm 2.5^{\mathrm{c}}$ & $25.28 \pm 2.9^{c}$ & $31.09 \pm 1.7^{\mathrm{c}}$ & $27.84^{\mathrm{c}}$ \\
\hline R.D & $75.85 \pm 2.9^{a}$ & $76.57 \pm 5.3^{\mathrm{a}}$ & $73.42 \pm 3.6^{\mathrm{a}}$ & $75.28^{\mathrm{a}}$ & $71.95 \pm 3.6^{\mathrm{a}}$ & $68.04 \pm 3.8^{\mathrm{a}}$ & $68.85 \pm 3.1^{\mathrm{a}}$ & $69.61^{\mathrm{a}}$ \\
\hline Average & $53.38^{\mathrm{a}}$ & $53.33^{\mathrm{a}}$ & $53.89^{\mathrm{a}}$ & & $54.08^{\mathrm{a}}$ & $52.14^{\mathrm{a}}$ & $51.42^{\mathrm{a}}$ & \\
\hline \multicolumn{9}{|c|}{ Flower bud diameter (mm) } \\
\hline R.B. & $9.79 \pm 0.12^{\mathrm{fg}}$ & $9.92 \pm 0.11^{\text {ef }}$ & $10.23 \pm 0.11^{\text {def }}$ & $9.98^{c}$ & $10.08 \pm 0.12^{\mathrm{cd}}$ & $9.74 \pm 0.09^{\mathrm{de}}$ & $10.35 \pm 0.14^{\mathrm{c}}$ & $10.06^{\mathrm{b}}$ \\
\hline R.C & $10.22 \pm 0.10^{\text {def }}$ & $10.87 \pm 0.16^{\mathrm{c}}$ & $10.27 \pm 0.13^{\mathrm{de}}$ & $10.45^{\mathrm{b}}$ & $9.4 \pm 0.19^{\mathrm{ef}}$ & $11.25 \pm 0.13^{\mathrm{b}}$ & $10.18 \pm 0.13^{\mathrm{cd}}$ & $10.28^{\mathrm{b}}$ \\
\hline G.T & $11.79 \pm 0.13^{\mathrm{b}}$ & $11.85 \pm 0.15^{\mathrm{b}}$ & $12.55 \pm 0.26^{\mathrm{a}}$ & $12.06^{\mathrm{a}}$ & $11.65 \pm 0.15^{b}$ & $12.35 \pm 0.16^{\mathrm{a}}$ & $12.5 \pm 0.24^{\mathrm{a}}$ & $12.17^{\mathrm{a}}$ \\
\hline R.D & $10.09 \pm 0.10^{\mathrm{def}}$ & $10.39 \pm 0.16^{\mathrm{d}}$ & $9.37 \pm 0.12^{\mathrm{g}}$ & $9.95^{\mathrm{c}}$ & $9.58 \pm 0.12^{\mathrm{e}}$ & $9.72 \pm 0.17^{\mathrm{de}}$ & $8.98 \pm 0.18^{\mathrm{f}}$ & $9.43^{\mathrm{c}}$ \\
\hline Average & $10.47^{\mathrm{b}}$ & $10.76^{\mathrm{a}}$ & $10.60^{\mathrm{ab}}$ & & $10.18^{\mathrm{c}}$ & $10.76^{\mathrm{a}}$ & $10.50^{\mathrm{b}}$ & \\
\hline \multicolumn{9}{|c|}{ Number of flowers per plant } \\
\hline R.B. & $86.33 \pm 5.6^{\mathrm{e}}$ & $90.47 \pm 4.8^{\mathrm{e}}$ & $81.93 \pm 5.1^{\mathrm{e}}$ & $86.24^{\mathrm{c}}$ & $94.56 \pm 5.4^{\mathrm{d}}$ & $96.89 \pm 7.3^{\mathrm{d}}$ & $91.22 \pm 6.6^{\mathrm{d}}$ & $94.22^{\mathrm{c}}$ \\
\hline R.C & $328.4 \pm 28.4^{\mathrm{cd}}$ & $367.27 \pm 25.9^{c}$ & $296.8 \pm 18.1^{\mathrm{d}}$ & $330.82^{b}$ & $342.44 \pm 15.5^{\mathrm{b}}$ & $368.56 \pm 19.6^{\mathrm{b}}$ & $283.56 \pm 8.9^{c}$ & $331.52^{\mathrm{b}}$ \\
\hline G.T & $473.73 \pm 25.5^{\mathrm{b}}$ & $535.67 \pm 20.8^{\mathrm{a}}$ & $456.07 \pm 17.4^{\mathrm{b}}$ & $488.49^{\mathrm{a}}$ & $508.56 \pm 18.9^{\mathrm{a}}$ & $561.67 \pm 15.1^{\mathrm{a}}$ & $523.89 \pm 16.0^{\mathrm{a}}$ & $531.37^{\mathrm{a}}$ \\
\hline R.D & $87.0 \pm 6.4^{\mathrm{e}}$ & $97.2 \pm 6.2^{\mathrm{e}}$ & $84.67 \pm 7.1^{\mathrm{e}}$ & $89.62^{c}$ & $88.11 \pm 8.2^{\mathrm{d}}$ & $97.56 \pm 8.6^{\mathrm{d}}$ & $83.56 \pm 5.1^{\mathrm{d}}$ & $89.74^{\mathrm{c}}$ \\
\hline Average & $243.86^{\mathrm{b}}$ & $272.65^{\mathrm{a}}$ & $229.86^{b}$ & & $258.42^{\mathrm{ab}}$ & $281.17^{\mathrm{a}}$ & $245.56^{\mathrm{b}}$ & \\
\hline \multicolumn{9}{|c|}{ Flower diameter $(\mathrm{mm})$} \\
\hline R.B. & $45.35 \pm 0.9^{\text {cd }}$ & $45.10 \pm 0.8^{\mathrm{d}}$ & $45.97 \pm 1.2^{\text {cd }}$ & $45.48^{b}$ & $43.94 \pm 0.6^{\mathrm{i}}$ & $48.40 \pm 0.9^{\text {ef }}$ & $44.53 \pm 0.9^{\mathrm{hi}}$ & $45.62^{\mathrm{c}}$ \\
\hline R.C & $53.86 \pm 1.1^{\mathrm{b}}$ & $45.77 \pm 0.7^{\mathrm{cd}}$ & $41.38 \pm 0.9^{\mathrm{e}}$ & $47.01^{\mathrm{b}}$ & $51.56 \pm 0.9^{c}$ & $46.61 \pm 0.5^{\mathrm{fg}}$ & $42.64 \pm 0.4^{\mathrm{i}}$ & $46.94^{\mathrm{b}}$ \\
\hline G.T & $52.40 \pm 1.3^{\mathrm{b}}$ & $62.76 \pm 1.2^{\mathrm{a}}$ & $59.96 \pm 1.6^{\mathrm{a}}$ & $58.37^{\mathrm{a}}$ & $50.85 \pm 0.8^{\mathrm{cd}}$ & $58.48 \pm 0.5^{\mathrm{a}}$ & $55.47 \pm 0.9^{\mathrm{b}}$ & $54.93^{\mathrm{a}}$ \\
\hline R.D & $48.39 \pm 1.1^{\mathrm{c}}$ & $44.45 \pm 0.6^{\mathrm{d}}$ & $44.22 \pm 0.6^{\mathrm{de}}$ & $45.69^{b}$ & $49.40 \pm 0.6^{\mathrm{de}}$ & $46.63 \pm 0.5^{\mathrm{fg}}$ & $46.13 \pm 0.6^{\mathrm{gh}}$ & $47.39^{\mathrm{b}}$ \\
\hline Average & $50.00^{\mathrm{a}}$ & $49.52^{\mathrm{a}}$ & $47.88^{\mathrm{b}}$ & & $48.94^{\mathrm{ab}}$ & $49.27^{\mathrm{a}}$ & $47.95^{\mathrm{b}}$ & \\
\hline \multicolumn{9}{|c|}{ Number of petals per flower } \\
\hline R.B. & $45.18 \pm 1.4^{\mathrm{ab}}$ & $45.76 \pm 1.3^{\mathrm{ab}}$ & $42.04 \pm 0.7^{\mathrm{d}}$ & $44.50^{\mathrm{a}}$ & $42.52 \pm 1.1^{\mathrm{cd}}$ & $45.66 \pm 0.7^{\mathrm{a}}$ & $44.47 \pm 1.1^{\mathrm{ab}}$ & $44.11^{\mathrm{b}}$ \\
\hline R.C & $42.42 \pm 0.7^{\mathrm{cd}}$ & $45.19 \pm 1.2^{\mathrm{ab}}$ & $40.71 \pm 0.4^{\mathrm{d}}$ & $42.77^{\mathrm{b}}$ & $43.38 \pm 0.9^{\mathrm{bcd}}$ & $45.57 \pm 0.5^{\mathrm{ab}}$ & $41.47 \pm 0.6^{\mathrm{d}}$ & $43.47^{\mathrm{b}}$ \\
\hline G.T & $44.90 \pm 0.7^{\mathrm{abc}}$ & $46.31 \pm 1.0^{\mathrm{a}}$ & $45.19 \pm 0.5^{\mathrm{ab}}$ & $45.28^{\mathrm{a}}$ & $44.85 \pm 0.3^{\mathrm{ab}}$ & $46.28 \pm 0.4^{\mathrm{a}}$ & $45.81 \pm 0.4^{\mathrm{a}}$ & $45.65^{\mathrm{a}}$ \\
\hline R.D & $41.52 \pm 0.6^{\mathrm{d}}$ & $43.04 \pm 0.6^{\mathrm{bcd}}$ & $42.42 \pm 0.4^{\mathrm{cd}}$ & $42.33^{\mathrm{b}}$ & $41.41 \pm 0.5^{\mathrm{d}}$ & $42.09 \pm 0.8^{\mathrm{d}}$ & $42.0 \pm 0.9^{\mathrm{d}}$ & $41.83^{\mathrm{c}}$ \\
\hline Average & $43.50^{\mathrm{b}}$ & $45.06^{\mathrm{a}}$ & $42.59^{\mathrm{b}}$ & & $43.04^{\mathrm{b}}$ & $44.90^{\mathrm{a}}$ & $43.44^{\mathrm{b}}$ & \\
\hline
\end{tabular}

Treatments sharing similar statistical letters are significantly not different from each other. $\mathrm{CW}=\mathrm{Canal}$ water; TW= Treated waste water; UTW= Untreated waste water. $\mathrm{RB}=$ Rosa bourboniana $; \mathrm{RC}=$ Rosa centifolia $; \mathrm{GT}=$ Gruss-an-Teplitz; RD= Rosa damascena . 
water treatment whereas maximum value was recorded for $R$. Gruss-an-Teplitz $(12.50 \mathrm{~mm})$ under untreated waste water (Table 4).

Number of flowers plant ${ }^{-1}$ : The results revealed that $R$. Grussan-Teplitz (535.67) under treated waste water produced highest number of flowers plant ${ }^{-1}$ in 2012 whereas $R$. bourboniana (81.93) under untreated waste water produced minimum number of flowers. During $1^{\text {st }}$ year of the experiment, $R$. centifolia and $R$. damascena produced 367.27 and 97.20 flowers respectively under treated waste water treatment and these values were at top in respective species among all irrigation treatments. In 2013, $R$. Gruss-an-Teplitz (561.67) produced maximum and $R$. damascena (83.56) produced minimum number of flower plant $^{-1}$ year $^{-1}$ under treated waste water and untreated waste water treatment respectively. In $R$. centifolia, flower quantity was reduced from 367.27 to 296.80 from treated to untreated waste water respectively while in $R$. bourboniana (96.89) maximum flowers were produced under treated waste water (Table 4).

Flower diameter $(\mathbf{m m})$ : Data showed that highest value of flower diameter was recorded in $R$. Gruss-an-Teplitz $(62.76 \mathrm{~mm})$ under treated waste water during 2012 while $R$. centifolia produced minimum value of flower diameter (41.38mm) under untreated waste water. During 2013, $R$. Gruss-an-Teplitz $(58.48 \mathrm{~mm})$ under treated waste water produced maximum flower diameter whereas $R$. centifolia $(42.64 \mathrm{~mm})$ in same irrigation treatment obtained minimum value (Table 4).

Number of petals flower ${ }^{-1}$ : The highest value of flower petals was found in $R$. Gruss-an-Teplitz (46.31) followed by $R$. bourboniana (45.76) under treated waste water treatment during 2012 whereas minimum number of petals (40.71) was recorded in $R$. centifolia under untreated waste water treatment. In 2013, maximum petal numbers was produced in $R$. Gruss-an-Teplitz (46.28) under treated waste water treatment while minimum values were recorded in $R$. damascena (41.41) under treated waste water treatment (Table 4).

\section{DISCUSSION}

Water used in this experiment was basic in nature as its $\mathrm{pH}$ was more than 7 and EC of untreated waste water was more than standard values set by international irrigation water quality standards (IIWQS) and national environmental quality standards (NEQS) for municipal waste waters of Pakistan. All other minerals and chemicals under treated waste water and canal water treatment were in permissible range. Untreated waste water contained higher concentration of some toxic heavy metals (i.e. $\mathrm{Cd}, \mathrm{Pb}, \mathrm{Co}, \mathrm{Cu}$ ) and for this reason its BOD and COD were high (Kakar et al., 2011). Plants were silent sufferers, so their response against untreated waste water was reduced growth and lesser flower yield as compare to canal water and treated waste water treatments.
Fragrant Rosa species differed significantly for plant height in waste water irrigation. $R$. bourboniana seems to be most resistant species to pollutants in irrigation water as compare to others Rosa species and $R$. centifolia was least resistant. In all Rosa species, maximum height was recorded in canal water treatment which showed that waste water treatment has negative effect on plant height but in $R$. bourboniana, height was less affected under treated and untreated waste water. Effects of toxicity in irrigation water differ greatly from plant to plant but in some medicinal plants, toxicity in irrigation water does not alters/reduced the height (Bernstein et al., 2009). These results verified the findings of Younis (2006) who also reported that $R$. bourboniana produced maximum height than other oil bearing Rosa species in Pakistan. Results of this experiment were also in line with the findings of Sridhar et al. (2005) as increased in toxicity in irrigation water plant height was reduced depending upon plant species. Andleeb et al. (2008) also verified results of this study and stated that increase in metal concentration (especially $\mathrm{Cr}$ ) tends to decrease plant height in sunflower.

Singh and Agrawal (2010) and Sinha et al. (2007) observed significantly higher number of leaves in plants, irrigated by municipal waste water as compare to canal water irrigated plants. Results of present study also showed that there was more number of leaves under treated and untreated waste water treatment in all Rosa species. Findings of Aldesuquy (2014) supported the results of this study who argued that as load of heavy metals increases in irrigation water, leaf area in plants reduced but this character depends on plant type. Pandey and Tripathi (2011) also reported that concentration of heavy metals adversely affect the leaf area. Singh and Agrawal (2010) showed positive effects of waste water regarding leaf area and Rusan et al. (2008) reported that $R$. indica and $R$. canina showed highest leaf area in treated waste water treatment as compare to canal/fresh water.

It is the specific character of different Rosa species to produce flowers during different months of the year as $R$. centifolia and $R$. Gruss-an-Teplitz produced flowering during whole year while $R$. bourboniana and $R$. damascena produced flowers only in the months of March and April (Younis, 2006). I this study floral bud and flower diameter of $R$. bourboniana and $R$. Gruss-an-Teplitz were higher under treated and untreated waste water treatment as compare to canal water irrigation while this trend was opposite in case of $R$. centifolia and $R$. damascena. These results were comparable with the results of Rusan et al. (2008) and Marinho et al. (2013) who argued that due to availability of nutrients in treated and untreated waste water, flower buds and flower size of Rosa species responded positively and their diameter was higher as compare to flowers of fresh water treatment. These findings were contradictory to the results of Bernstein et al. (2009) who showed no effects of waste water on morphological attributes i.e. plant height, leaf area, flower bud diameter, flower size etc. in aromatic plant species. The 
increase in flower numbers per plant of $R$. Gruss-an-Teplitz in this study under waste water treatments over canal water could also be credited to the presence of high organic matter in waste water that can improve soil structure and availability of nutrients (Brady and Weil, 2008). The production limitations of Rosa species other than $R$. Gruss-an-Teplitz under waste water treatments might be instigated by higher EC of polluted irrigation treatments. Similar effects of high EC waters have been mentioned by different authors for olive (Ben Ahmed et al., 2008; Melgar et al., 2008). These results were contradictory with the results of Friedman et al. (2007) who reported that waste water has no effect on flower number. Darvishi et al. (2010) found that there was an increment in number of flowers per plant by the application of domestically treated waste water.

In this study, there was not any remarkable difference among Rosa species for number of petals per flowers but some petals were produced in $R$. centifolia and $R$. damascena that were small in size and malformed in shape and were not considered as petals under treated and untreated waste water treatment. These findings were according to the results of Nirit et al. (2006) who found no overall effect on flower development and shape but these results were contradictory with the findings of Khan et al. (2011) who showed increment in rose flower petal numbers in waste water irrigation treatment.

Conclusion: From this study it was concluded that under treated waste water, all morphological attributes of Rosa species showed the maximum response and most of the characteristics were improved significantly. The increase in the floral characteristics was highly prominent in $R$. Grussan-Teplitz such as minimum days to produce flowers, maximum bud and flower diameter, number of flowers per plant and number of petals per flower whereas, plant height and number of leaves were maximum in $R$. bourboniana but leaf area recorded in $R$. damascena was highly increased. In contrast to treated waste water, untreated waste water, reduced the values of all species where most of the characteristics showed decreasing trend. The overall impact of high concentration of metals and other contaminants was more severe in $R$. centifolia whereas $R$. Gruss-an-Teplitz was least affected and best performed species under untreated waste water.

Acknowledgements: The authors are very thankful to higher Education Commission (HEC), Government of Pakistan for providing generous funds for this study.

\section{REFERENCES}

Abu Qdais, H. and M. Al-Widyan. 2016. Evaluating composting and cocomposting kinetics of various agroindustrial wastes. Int. J. Recycle Org. Waste Agri. 5:273280 .
Aldesuquy, H.S. 2014. Effect of spermine and spermidine on wheat plants irrigated with waste water: Conductive canals of flag leaf and peduncle in relation to grain yield. J. Stress Physiol. Biochem. 10:145-166.

Alloway. B.J. 1990. Heavy metals in soils. Blackie Glasgow and London. John Wiley \& Sons. Inc. New York.

Andleeb, F., M.A. Zia, M. Ashraf and Z.M. Khalid. 2008. Effect of chromium on growth attributes in sunflower (Helianthus annuus L.). J. Environ. Sci. 20:1475-1480.

Anonymous. 2007. Water quality standards. Environmental Protection Agency. Government of Pakistan, Islamabad, Pakistan.

Asgharipour, M.R. and H.R. Azizmoghaddam. 2012. Effects of raw and diluted municipal sewage effluent with micronutrient foliar sprays on the growth and nutrient concentration of foxtail millet in southeast Iran. Saudi J. Biol. Sci. 19:441-449.

Ben Ahmed, C., B.B. Rouina and M. Boukhris. 2008. Changes in water relation, photosynthetic activity and proline accumulation in one year old tree (Olea europaea L. cv Chemlali) in response to $\mathrm{NaCl}$ salinity. Acta Physiol. Plant. 30:553-560.

Bernstein, N., D. Chaimovitch and N. Dudai. 2009. Effect of irrigation with secondary treated effluent on essential oil, antioxidant activity and phenolic compounds in oregano and rosemary. Agron. J. 101:1-10.

Brady, N.C. and R.R. Weil. 2008. The nature and properties of soils. $14^{\text {th }}$ ed. Pearson Education Inc., New Jersey.

Darvishi, H.H., M. Manshouri, H. Sedghi and S.H.M. Jahromi. 2010. Irrigation influence by treated domestic wastewater instead of agronomical water on essential oil yield of basil (Ocimum basilicum L.). Afr. J. Microbiol. Res. 4:475-479.

Eaton, A.D., L.S. Glescer, E.W. Rice and A.E. Greenberg. 2005. Standard methods for the examination of water and wastewater. $21^{\text {st }}$ ed. American Public Health Association. Washington, USA.

Farahat, E. and H.W. Linderholm. 2013. Effects of treated waste water irrigation on size-structure, biochemical products and mineral content of native medicinal shrubs. Ecol. Eng. 60:235-241.

Farooq, A., S. Lei, M. Nadeem, M. Asif, G. Akhtar and S.J. Butt. 2016. Cross compatibility in various scented rosa species breeding. Pak. J. Agri. Sci. 53:863-869.

Friedman, H., N. Bernstein, M. Bruner, I. Rot, Z. Ben-Noon, A. Zuriel, R. Zuriel, S. Finkelstein, N. Umiel and A. Hagiladi. 2007. Application of secondary-treated effluents for cultivation of sunflower (Helianthus annuus L.) and celosia (Celosia argentea L.) as cut flowers. Sci. Hortic. 115:62-69.

Hussain, S.I., A. Ghafoor, S. Ahmad, G. Murtaza and M. Sabir. 2006. Irrigation of crops with raw sewage: hazard and assessment in effluent, soil and vegetables. Pak. J. Agric. Sci. 43:97-101. 
Janko, A.M. and G. Alemu. 2014. Supply and marketing of flowers in Ethopia. Unique J. Agric. Eco. Rural Dev. 1:924.

Kakar, S.R., A. Wahid, R.B. Tareen, S.A. Kakar, M. Tariq and S.A. Kayani. 2011. Impact of municipal waste water of Quetta city on biomass, physiology and yield of canola (Brassica napus L.). Pak. J. Bot. 42:317-328.

Khaleel, R.I., N. Ismail and M.H. Ibrahim. 2013. The impact of wastewater treatments on seed germination and biochemical parameter of Abelmoschus esculentus L. Soc. Behav. Sci. 91:453-460.

Khan, M.A., S.S. Shaukat, A. Shahzad and W. Ahmed. 2011. Application of waste stabilization pond's effluent on cultivation of roses (Rosa damascena Mill). Pak. J. Bot. 43:1919-1923.

Kiziloglu, F.M., M. Turan, U. Sahin, Y. Kuslu and A. Dursun. 2008. Effects of untreated and treated wastewater irrigation on some chemical properties of cauliflower (Brassica olerecea L. var. Botrytis) and red cabbage (Brassica olerecea L. var. Rubra) grown on calcareous soil in Turkey. Agric. Water Manage. 95:716-724.

Kurian, M. 2017. The water-energy-food nexus: trade-offs, thresholds and transdisciplinary approaches to sustainable development. Environ. Sci. Policy 68:97106.

Laurie, A. and V.H. Ries. 1950. Floriculture: Fundamentals and Practices, $2^{\text {nd }}$ Ed. McGraw Hill Book Co., New York.

Marinho, L.E.O., A.L. Tonetti, R. Stefanutti and B.C. Filho. 2013. Application of reclaimed wastewater in the irrigation of rosebushes. Water Air Soil Poll. 224:16691675.

Melgar, J.C., J.P. Syversten and F. Garcia-Sanchez. 2008. Can elevated $\mathrm{CO}_{2}$ improve salt tolerance in olive trees. J. Plant Physiol. 165:631-640.

Murtaza, G., A. Ghafoor, M. Qadir, G. Owens, M.A. Aziz, M.H. Zia and Saifullah. 2010. Disposal and use of sewage on agricultural lands in Pakistan: A review. Pedosphere 20:23-34.

Nasir, M.H., R. Nadeem, K. Akhtar, M.A. Hanif and A.M. Khalid. 2007. Efficacy of modified distillation sludge of rose (Rosa centifolia) petals for lead (II) and zinc (II) removal from aqueous solutions. J. Hazard Mater. 147:1006-1014.

Nirit, B., B.T. Asher, F. Haya, S. Pini, R. Ilona, C. Amram and I. Marina. 2006. Application of treated wastewater for cultivation of roses (Rosa hybrida) in soilless culture. Sci. Hortic. 108:185-193.

Pandey, P. and A.K Tripathi. 2011. Effect of heavy metals on morphological and biochemical characteristics of Albizia procera (Roxb.) Benth. seedlings. Int. J. Environ. Sci. 5:1009-1018.

Pescod, M.B. 1992. Wastewater treatment and use in agriculture. Irrig. Drain. 47, FAO, Rome.

Riaz, A., M. Arshad, A. Younis, A. Raza and M. Hameed. 2008. Effect of different growing media on growth and flowering of Zinnia elegance Blue point. Pak. J. Bot. 40:1579-1585.

Rusan, M.J.M., I. Bashabsheh and M. Safi. 2008. Reuse of treated wastewater for cut flowers production and impact on soil and plant quality parameters. Int. Conf. Const. Build. Technol. 6:63-78.

Singh, A. and M. Agrawal. 2010. Effects of municipal waste water irrigation on availability of heavy metals and morpho-physiological characteristics of Beta vulgaris L. J. Environ. Biol. 31:727-736.

Sinha, S., S. Mallick, R.K. Misra, S. Singh, A. Basant and A.K. Gupta. 2007. Uptake and translocation of metals in Spinacia oleracea L. grown on tannery sludge-amended and contaminated soils: Effect on lipid peroxidation, morpho-anatomical changes and antioxidants. Chemosphere 67:176-187.

Sridhar, B.B.M., S.V. Diehl, F.X. Hanc, D.L. Monts and Y. Sub. 2005. Anatomical changes due to uptake and accumulation of $\mathrm{Zn}$ and $\mathrm{Cd}$ in Indian mustard (Brassica juncea). Environ. Exp. Bot. 54:131-141.

Steel, R.G.D., J.H Torrie and D.A Dickey. 1997. Principles and Procedures of Statistics: A biometric approach, $3^{\text {rd }}$ Ed. McGraw Hill Book Co., New York.

Talal, A., I. Ismail, J.M. Basahi, A. Qari and I.A. Hassan. 2014. Hazardous of waste water irrigation on quality attributes and contamination of citrus fruits. Biosci. Biotechnol. Res. Asia 11:89-97.

U.S. Salinity Lab. Staff. 1954. Diagnosis and improvement of saline and alkali soils. USDA Hand Book No. 60. Washington. D.C., U.S.A.

WHO. 1989. Health guidelines for the use of waste water in agriculture and aquaculture. WHO Technical Report Series 778. Geneva, Switzerland.

Younis, A. 2006. Phytochemical analysis and potential for exploitation of heterosis for essential oil contents in Rosa species. Ph.D. Diss. Uni. Agric. Faisalabad, Pakistan. 